\title{
Building Narrative Structures Using Context Based Linking
}

\author{
Mark J. Weal, David E. Millard, Danius T. Michaelides, David C. De Roure \\ IAM Group \\ Department of Electronics \& Computer Science \\ University of Southampton \\ SO17 1BJ, UK \\ phone $+44(0) 2380594059$ \\ $\{m j w, d e m, d t m, d d e r\} @$ ecs.soton.ac.uk
}

\begin{abstract}
This paper discusses initial progress in the construction of a hypertext short fiction engine using a context based link service. The link service, Auld Leaky, is based around the Fundamental Open Hypermedia Model (FOHM). Context and behaviour are used to provide adaption in the story as well as progressing the narrative.
\end{abstract}

KEYWORDS: Open Hypermedia, Fundamental Open Hypermedia Model (FOHM), Context, Adaptive Hypermedia, Narrative.

\section{INTRODUCTION}

When hypertext fiction is discussed in the literature, two of the most commonly referenced pieces are 'Afternoon, a story', by Michael Joyce [3] and 'Victory Garden', by Stuart Moulthrop [5]. Both of these are very different from conventional linear narrative. Perhaps the most obvious reason why this is true is the use of repetition and the revisiting of nodes to re-interpret them in a new light [7]. This often reduces the role of narrative, providing an experience more akin to that of reading poetry [6]. The fragments slowly build up an overall picture for the reader, where the chronology is often non-linear, making use of both flashback and analeptic and proleptic transitions between nodes [1].

An alternative to this is the branching novel, where an explicit narrative structure leads the reader through the story with the reader choosing to follow different branches at various points along the way.

By using a context based link service, this work aims to enable a hybrid of these two approaches, combining the fluid, loosely structured story fragment approach, with an overlayed narrative structure.

\section{A FOHM BASED LINK SERVICE}

This work uses a new link service called Auld Leaky, which places context at the heart of its matching algorithms. Auld Leaky is constructed around the Fundamental Open Hypertext Model (FOHM) [4]. This model grew out of the Open Hypermedia Protocol (OHP) developed by the Open Hypermedia Systems Working Group OHSWG) [2].

The basic FOHM model is constructed from four core objects. These are :-

- Associations, representing relationships between data objects (or other associations).

- Data objects, which serve as wrappers for any piece of data held outside of the model.

- References, which are used to point at data objects and associations.

- Bindings, which are used to attach the references to the association structure.

Figure 1 shows a basic navigational link which has a source and two destinations.

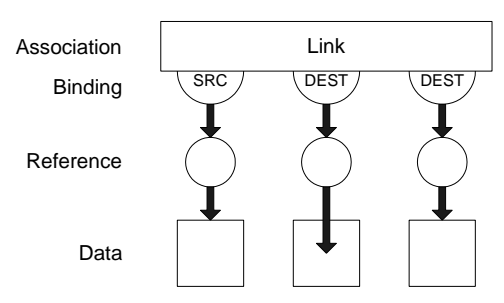

Figure 1: A navigational link in FOHM.

The FOHM model allows context and behaviour to be attached at any point on the FOHM structure. Context is used in the matching process, with links only being returned when the contexts of the query and the target structures match. Behaviour is opaque to the Auld Leaky implementation, and is interpreted by the client. It allows hypermedia authors to specify how certain events, such as 'follow link', alter the users context, e.g. progressing them through a timeline. Examples of how context and behaviour can be used in the construction of stories are given in the section below. 


\section{HYPERTEXT SHORT STORIES}

The FOHM structures and Auld Leaky can be used as a mechanism to create adaptive hypermedia short stories. A web based client has been constructed that builds the stories for the reader on the fly from story fragments held in a linkbase.

Story fragments are data objects, containing a piece of the story. A data object can have behaviour attached to it, which is used by the client to modify the readers context when the data object is displayed.

To link all of these story fragments together, associations are authored within the linkbase. These associations are unlike standard navigational links in that their source binding is generic, i.e. not bound to a specific reference. Instead, the association has a context attached to it. The client constructing the story queries the linkbase with a user context and is returned all of the associations that match in that user context. Figure 2. shows the association structure used in the application.

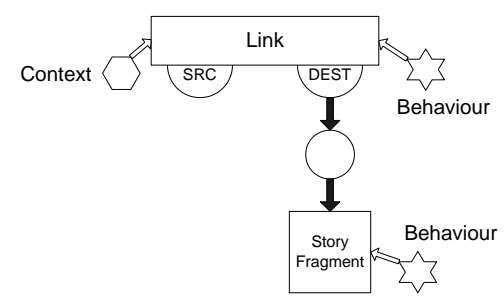

\section{Figure 2: A context source link in FOHM}

Links can also have behaviour attached to them. The client interprets the link behaviour when it traverses the link, i.e. when the reader clicks on the link to read the next story fragment.

The context and behaviour can be used in a number of different ways:-

- To maintain a record of time. For example, this allows certain story fragments to be triggered at specific times.

- To keep note of character location. Story fragments are scoped to the current location.

- To keep a record of key events that happen in the story. This allows for the foreshadowing of later events.

\section{Narrative Structure}

Context can also be used to keep track of where the reader is in the overall plot of the story. For example, the plot could be written with a three act structure. This aspect of the context can then be used to restrict basic exposition and background information to the beginning of the story where the scene is being set. Once the reader gets into the heart of the story, superfluous atmosphere story fragments are no longer offered to them as at this stage they may just interrupt the flow of the narrative. This is just one way in which the narrative context can be used to steer the reader through the story. Other, more complicated plot structures, could also be envisaged.

\section{CONCLUSIONS AND FUTURE WORK}

The aim of this work is to explore the possibilities of using a contextual link service to author hypertext short fiction. Our current examples use context and behaviour to maintain a logical path through the story chronology, incorporating location and event information. We intend to improve on the simple implicit narrative structures we have been using so far and experiment with more complex structures.

Because the narrative structure is held within the links, different narrative structures can be placed over the same story fragments, allowing them to be re-used in different stories.

The authoring process allows the author to concentrate on the story fragments as elements without requiring them to explicitly wire them together with links. There is clearly scope for the creation of tools to aid in the authoring process, particularly as implicitely coupled story fragments exhibit some traditional hypertext problems such as dead-ends and unreachable nodes.

We have not gone into detail on what constitutes context in our systems and indeed this is the subject of ongoing work. At the moment key-value pairs are sufficient, but we are investigating ontologies that can be used to reason about context in more complex ways.

Examples of our experiments in this area can be found at:

http://www.equator.ecs.soton.ac.uk/hyperfiction/

\section{ACKNOWLEDGEMENTS}

This research is funded in part by EPSRC IRC project "EQUATOR" GR/N15986/01 and by EPSRC DIM project "LinkMe" GR/M25919

\section{REFERENCES}

1. CAlvi, L. 'Lector in rebus': The Role of the Reader and the Characteristics of Hyperreading. In Hypertext '99, Darmstadt, Germany. (Feb. 1999).

2. Davis, H. C., Millard, D. E., Reich, S., Bouvin, N., GrønbÆK, K., NÜRnberG, P. J., Sloth, L., WiIl, U. K., AND ANDERSON, K. M. Interoperability between hypermedia systems: The standardisation work of the ohswg. In Hypertext '99, The 10th ACM Conference on Hypertext and Hypermedia, Darmstadt (Feb. 1999), ACM, pp. 201-202.

3. JoyCe, M. Afternoon, a story. Eastgate Systems, 1987.

4. Millard, D. E., Moreau, L., Davis, H. C., and Reich, S. FOHM: A Fundamental Open Hypertext Model for Investigating Interoperability Between Hypertext Domains. In Proceedings of the 'OO ACM Conference on Hypertext, May 30 June 3, San Antonio, TX (2000), pp. 93-102.

5. Moulthrop, S. Victory Garden. Eastgate Systems, 1991.

6. ToscA, S. A. The Lyrical Quality of Links. In Hypertext '99, Darmstadt, Germany. (Feb. 1999).

7. WALker, J. Piecing together and tearing apart: finding the story in afternoon. In Hypertext '99, Darmstadt, Germany. (Feb. 1999). 